\title{
Adaptação da Escala de Discriminação Quotidiana para Jovens Portugueses
}

\author{
Adaptation of the Everyday Discrimination Scale to Portuguese Youth
}

\author{
Daniela Fonseca Freitas ${ }^{*}, a$, Susana Coimbra ${ }^{a}$, Edna Maria Marturano ${ }^{b} \&$ Anne Marie Fontaine ${ }^{a}$ \\ ${ }^{a}$ Universidade do Porto, Porto, Portugal \& ${ }^{b}$ Universidade de São Paulo, Ribeirão Preto, SP, Brasil
}

\begin{abstract}
Resumo
O objetivo deste estudo foi a adaptação da Escala de Discriminação Quotidiana para a população jovem portuguesa. Após tradução, ajustes semânticos aos itens, inclusão de dois itens para aumento da validade de constructo e a aferição da validade facial, os itens da escala foram respondidos por 2996 jovens estudantes do ensino secundário em Portugal. A Análise Fatorial Exploratória (AFE) revelou uma estrutura de dois fatores - Tratamento Injusto e Rejeição Pessoal - com quatro itens cada (sete itens da escala original e um formulado neste estudo). A Análise Fatorial Confirmatória (AFC) validou um modelo hierárquico, em que os dois fatores observados na AFE estão subordinados a um fator latente de segunda ordem, a Percepção de Discriminação. A escala permite diferenciar a discriminação percepcionada por grupos alvo de preconceito social, observando-se diferenças significativas nos valores reportados entre pessoas negras e brancas e entre jovens lésbicas, gays, bissexuais e participantes heterossexuais. $\mathrm{O}$ instrumento explica parcialmente variações ao nível de saúde mental, observando-se uma correlação negativa moderada entre a discriminação percepcionada e a saúde mental. Os resultados sugerem que a versão adaptada da escala apresenta validade de constructo, sendo bastante apropriada para avaliar e comparar a percepção de discriminação de jovens. Palavras-chave: Validação, análise fatorial confirmatória, discriminação.
\end{abstract}

\begin{abstract}
The aim of this study was the adaptation of the Everyday Discrimination Scale to Portuguese youth. Scale items were subject to translation and adaptation, and two items were incorporated into the scale to increase construct validity. After establishing face validity, data from 2996 high school students were subject to Exploratory Factorial Analysis (EFA) and Confirmatory Factor Analysis (CFA). The EFA results portrayed a two-factor solution - Unfair Treatment and Personal Rejection - with four items each (seven items of the original scale and one prepared to this study). The CFA validated a hierarchical model in which the two factors observed in the EFA are subordinated to a second order latent factor, the Perception of Discrimination. The instrument was able to distinguish the perceptions of discrimination felt by prejudiced groups, since significant differences were observed between black and white participants, and between lesbian, gay and bisexual participants and their heterosexual counterparts. The instrument partially explains variations in mental health levels, given the observation of a negative correlation between perceived discrimination and mental health. The study concludes that this adapted version of the scale is a proper resource to the measurement and comparison of the perceptions of discrimination felt by the young.

Keywords: Validation, confirmatory factorial analysis, discrimination.
\end{abstract}

A percepção de discriminação define-se como a avaliação de uma ação como injusta ou imerecida, explicada pela pertença de uma pessoa a um grupo socialmente

\footnotetext{
"Endereço para correspondência: Universidade do Porto, Faculdade de Psicologia e de Ciências da Educação, Rua Alfredo Allen, Porto, Portugal 4200-135. E-mail: daniela. ffreitas@gmail.com e susana@fpce.up.pt

Este trabalho foi financiado por uma bolsa de doutoramento atribuída a Daniela Freitas pela FCT - Fundação para a Ciência e Tecnologia (SFRH/BD/79575/2011) e por uma da Universidade de São Paulo (USP) para alunos de Instituições de Ensino Superior Estrangeiras (Bolsa Internacional USP: 2014.1.3765.1.1) concedida à mesma pela autora.
}

estigmatizado (Major \& Sawyer, 2009). Considerando que este impacto negativo é também observado em outras situações de rejeição, Richman e Leary (2009) integram a discriminação num modelo mais abrangente de rejeição interpessoal, juntamente com o bullying, a estigmatização ou a humilhação. De uma forma global, a discriminação encontra-se associada a níveis mais baixos de desempenho académico e laboral, de saúde física e psicológica, bem como de autoestima e de satisfação com a vida (Krieger, 1999; Pascoe \& Richman, 2009; Schmitt, Branscombe, Postmes, \& Garcia, 2014; Williams \& Mohammed, 2009). A percepção de um tratamento injusto e de rejeição pessoal afiguram-se como mecanismos de risco incontornáveis 
para crianças, jovens e adultos. Assim, é importante avaliar em que medida as pessoas experienciam estas situações, os contextos onde ocorrem e as suas diversas manifestações, de forma a melhor informar os agentes de intervenção social e planear projetos de intervenção eficientes.

Para avaliar a percepção de discriminação, vários instrumentos têm sido desenvolvidos, sendo a Escala de Discriminação Quotidiana (Everyday Discrimination Scale; Williams, Yu, Jackson, \& Anderson, 1997) um dos mais utilizados (Krieger, 1999; Pascoe \& Richman, 2009; Williams \& Mohammed, 2009). Este instrumento foi desenvolvido, em 1995, nos EUA (Williams et al., 1997). Os itens tiveram por base estudos qualitativos junto de mulheres negras Americanas e Holandesas, realizados por Essed (1990 \& 1991, como citado por Kim, Sellbom, \& Ford, 2014; \& Lewis, Yang, Jacobs, \& Fitchett, 2012; e por Williams et al., 1997) e visavam capturar pequenos atos de tratamento injusto que ocorrem rotineiramente $\mathrm{e}$ que são passíveis de ser vivenciados por qualquer pessoa. Estes pequenos atos, também designados de discriminação encoberta, podem ocorrer de forma crónica e sistemática (Williams et al., 1997). Alguns estudos sugerem que a discriminação encoberta tem uma relação de maior magnitude com a perturbação psicológica (e.g., depressão, ansiedade) do que a discriminação aberta (como ser negado/a um emprego ou uma promoção; Taylor, Kamarck, \& Shiffman, 2004; Williams et al., 1997).

Williams et al. (1997) haviam estimado, quando da formulação dos itens, que as situações de tratamento injusto não seriam vividas apenas por pessoas negras. Não obstante, pelo que é do nosso conhecimento, a escala tem sido, preferencialmente, utilizada para a avaliação da discriminação racial e apenas um estudo procurou a sua validação em função do gênero (Stucky et al., 2011). Não é por isso de admirar que vários estudos de validação se foquem na aferição das suas propriedades psicométricas em grupos raciais/étnicos distintos (Barnes et al., 2004; Guyll, Matthews, \& Bromberger, 2001; Kim et al., 2014; Lewis et al., 2012). Estes estudos testaram as semelhanças estruturais na escala entre pessoas negras e brancas (Barnes et al., 2004; Guyll et al., 2001), bem como a variância na consideração de alguns itens em função da raça/etnia (Kim et al., 2014; Lewis et al., 2012) e do gênero (Stucky et al., 2011). Foi, ainda, feita uma recomendação ao uso de uma versão reduzida (de 5 itens) da escala (Stucky et al., 2011). Dado que estes estudos foram conduzidos com amostras provenientes dos EUA, é necessário o estudo das propriedades da escala em outras culturas. Não temos conhecimento de estudos que tenham utilizado este instrumento em Portugal.

No que concerne à estrutura da escala, grande parte dos estudos apontam uma organização unifatorial (Kessler, Mickelson, \& Williams, 1999; Kim et al., 2014; Krieger, Smith, Naishadham, Hartman, \& Barbeau, 2005; Seaton, Caldwell, Sellers, \& Jackson, 2010; Williams et al., 1997).
Porém, Barnes et al. (2004) e Guyll et al. (2001), identificaram uma estrutura bifatorial: no primeiro caso, designaram as subescalas de "tratamento injusto flagrante" (e.g., ser ameaçado) e "tratamento injusto subtil" (e.g., ser tratado com menos respeito) e no segundo de "tratamento injusto" (e.g., ser tratado com menos simpatia) e "rejeição pessoal" (e.g., ser insultado). Nestes estudos não é mencionada a existência de respostas diferenciais a cada subescala, em função de variáveis sociodemográficas.

A escala demonstrou validade convergente com inventários de situações importantes de discriminação ao longo da vida (e.g., ter sido injustamente recusado um emprego, promoção laboral ou empréstimo bancário; Kessler et al., 1999; Williams et al., 1997) - e de autorrelato de experiências de discriminação menores em contextos públicos (Krieger et al., 2005). Estes resultados sugerem que as experiências menores de discriminação, que ocorrem de forma mais camuflada, estão também associadas a situações de discriminação mais óbvias e condicionadoras da vida das pessoas. No que concerne à validade divergente, Taylor et al. (2004) demonstraram que os dados recolhidos com o instrumento não se encontravam relacionados com outras formas de estresse não social (e.g., a exigência laboral ou controlo na tomada de decisão), ou com características individuais, particularmente a hostilidade perante outros. Estes resultados sugerem que os itens da escala de percepção de discriminação quotidiana não avaliam uma maior vulnerabilidade ao estresse pessoal ou predisposição negativa nas relações sociais. Os processos de validação, convergente e divergente, apresentados suportam a precisão do instrumento em avaliar a percepção de discriminação.

Apesar da já referida predominância de estudos no domínio da discriminação étnica ou racial (Pascoe \& Richman, 2009), a escala tem a particularidade de avaliar o tratamento injusto sem o relacionar diretamente a algum tipo de preconceito. A investigação sugere que a percepção de preconceito e de discriminação, mais aberta ou mais subtil, pode estar relacionada com uma variedade de fatores, tais como o gênero, a orientação sexual, a raça e/ ou etnia e aspetos de aparência física (e.g., Schmitt et al., 2014). Na versão original do instrumento (Williams et al., 1997), os participantes têm que se pronunciar sobre a frequência com que experienciam nove situações de tratamento injusto. Adicionalmente, quando uma (ou várias) dessas situações ocorre $(\mathrm{m})$ várias vezes ao ano, os participantes têm que se pronunciar sobre qual "o principal motivo" a que atribuem essa(s) situação(ões), escolhendo-o a partir de uma lista de características (e.g., raça, gênero, orientação sexual, peso, entre outras). Assim, a Escala de Discriminação Quotidiana é adequada para avaliar a frequência de discriminação percepcionada não apenas em pessoas de um grupo alvo de um preconceito particular (e.g., racismo), mas em todas as pessoas que, potencialmente, possam ser vítimas de discriminação, com base em qualquer uma das características enunciadas. 
O objetivo deste artigo é apresentar a adaptação da Escala de Discriminação Quotidiana numa amostra de jovens portugueses, avaliando as suas propriedades psicométricas. Para os aspectos relativos à estrutura do instrumento foi realizada uma Análise Fatorial Exploratória (AFE), seguida de uma Análise Fatorial Confirmatória (AFC), e de procedimentos para aferir a consistência interna do instrumento. Para assegurar a validade de constructo aferido é crucial que o instrumento demostre sensibilidade para capturar variações no atributo avaliado e que a sua rede nomológica de relações seja congruente com a interpretação do fenómeno psicológico em questão (Borsboom, Mellenbergh, \& van Heerden, 2004). No que concerne a variações na percepção da discriminação, a literatura sugere que indivíduos com uma identidade social que são alvo de preconceito e/ ou que integram um grupo que é oprimido socialmente, sofrem mais discriminação do que indivíduos com uma identidade social dominante (e.g., indivíduos do género masculino, de ascendência europeia, com cor de pele clara, heterossexuais, de classe média ou alta e sem incapacidade física; Krieger, 1999). Deste modo, espera-se que um instrumento que avalia a percepção da discriminação seja capaz de capturar diferenças em função do estatuto social da pessoa, demostrando assim sensibilidade para apreender as variações neste atributo (Borsboom et al., 2004). Assim, de modo a providenciar evidência adicional para a validade de constructo, foram analisadas as diferenças de médias da percepção da discriminação em função da raça/ etnia e da orientação sexual. Com base nos estudos sobre a discriminação (e.g., Krieger, 1999; Pascoe \& Richman, 2009), previu-se que os participantes que se identificaram (a) como sendo de raça/etnia negra ou (b) com orientação sexual lésbica, gay ou bissexual (LGB) percepcionassem mais discriminação do que os (a) de raça/etnia branca/ caucasiana e (b) heterossexuais, respectivamente. Para reforçar a validade do instrumento é ainda fundamental que a sua rede nomológica de associações seja congruente com a interpretação teórica do fenómeno. O preconceito surge muitas vezes associado a comportamentos discriminatórios que aumentam a probabilidade de sentimento de desvalorização nas pessoas com uma característica estigmatizada socialmente (Goffman, 1963/1990). Por esse motivo, diversos estudos têm demostrado o impacto negativo da percepção de discriminação na saúde física, mental, e em dimensões do bem-estar, tais como a autoestima e a satisfação com a vida (e.g., Schmitt et al., 2014). Deste modo, no presente estudo, foi explorada a associação da escala adaptada com um instrumento de saúde mental, esperando encontrar uma correlação negativa entre estes dois instrumentos.

É importante mencionar que o termo raça usado neste trabalho não reflete uma perspectiva essencialista da diversidade humana, considerando-se antes que esta nomenclatura corresponde a um fenómeno socialmente construído. Reconhecemos que o uso desta categorização reforçou a perspectiva essencialista e poderá, ainda, continuar a fazê-lo (ver Smedley \& Smedley, 2005). Não obstante, consideramos que não poderemos abdicar deste conceito, uma vez que apenas pelo mapeamento destas identidades o racismo pode ser tornado visível e diminuído.

\section{Método}

\section{Participantes}

A amostra é constituída por 2996 jovens - com idades compreendidas entre os 13 e os 26 anos $(\mathrm{M}=16,6 ; \mathrm{DP}=$ 1,28 ) - estudantes do ensino secundário público obrigatório em Portugal, pertencentes a diferentes grupos sociais. A amostra é equilibrada em termos de gênero, a maioria dos participantes tem encarregados de educação com um nível de escolaridade acima da formação escolar obrigatória, mas sem formação no ensino superior, são brancos, católicos e heterossexuais. Considerando o âmbito da escala e o objetivo de adaptação e validação deste estudo, apenas os participantes que indicaram uma "característica-motivo" para o tratamento injusto (que foi vivenciado pelo menos algumas vezes por ano) foram selecionados $(\mathrm{n}=1999$, $66,9 \%$ ). Posteriormente, essa parte da amostra foi subdividida, de forma aleatória, em duas subamostras, sendo uma utilizada na análise fatorial exploratória e outra na análise fatorial confirmatória. Uma descrição detalhada da amostra total e das subamostras utilizadas, em ambas as análises, é apresentada na Tabela 1.

\section{Instrumentos}

Escala de Discriminação Quotidiana. A adaptação e a validação do instrumento incluiu diversos passos (Borsboom et al., 2004; Tanzer \& Sim, 1999). O segundo autor do estudo original do instrumento foi contactado de modo a autorizar o uso e a adaptação da escala. Os itens da Everyday Discrimination Scale foram traduzidos para a língua portuguesa pela primeira autora e a retroversão foi assegurada por uma segunda investigadora, com experiência de residência nos EUA e em Portugal. Os itens do instrumento foram alvo de reflexão por cinco investigadores nas áreas do preconceito, discriminação e desenvolvimento humano, e por três profissionais em associações comunitárias de pessoas com ascendência africana e de pessoas LGBT (lésbicas, gays, bissexuais e transgêneros) em Portugal. Dado que a discriminação tem por base os preconceitos (atitude negativa) e estereótipos (representações cognitivas) socialmente construídos sobre um grupo específico (Dovidio, Major, \& Crocker, 2000), e que havia a intenção de estudar também a discriminação associada à orientação sexual, incluíram-se itens que capturassem o preconceito homonegativo e a discriminação associada. Assim, de forma a aumentar a amplitude dos comportamentos avaliados e desse modo maximizar a validade ecológica para outros grupos alvo, como é o caso dos de orientação sexual minoritária, dois itens foram formulados com base na literatura (D'Augelli, Grossman, \& Starks, 2008; Gato, Carneiro, \& Fontaine, 2011) e na consulta a um profissional de uma associação 
Freitas, D. F., Coimbra, S., Marturano, E. M. \& Fontaine, A. M. (2015). Adaptação da Escala de Discriminação Quotidiana para Jovens Portugueses.

Tabela 1

Descrição Sociodemográfica dos Participantes do Estudo: Amostra Total, Amostra AEF e Amostra AFC

\begin{tabular}{|c|c|c|c|c|c|c|}
\hline & \multicolumn{2}{|c|}{$\begin{array}{c}\text { Amostra total } \\
N=2996\end{array}$} & \multicolumn{2}{|c|}{$\begin{array}{c}\text { Subamostra AFE } \\
n=985\end{array}$} & \multicolumn{2}{|c|}{$\begin{array}{c}\text { Subamostra AFC } \\
n=1013\end{array}$} \\
\hline & $N$ & $\%$ & $n$ & $\%$ & $n$ & $\%$ \\
\hline \multicolumn{7}{|l|}{ Sexo } \\
\hline Feminino & 1602 & 53,8 & 535 & 54,7 & 555 & 55,1 \\
\hline Masculino & 1373 & 46,2 & 443 & 45,3 & 453 & 44,9 \\
\hline \multicolumn{7}{|l|}{ Curso } \\
\hline Científico-humanístico & 2393 & 80,9 & 778 & 79,9 & 835 & 83,5 \\
\hline Profissional & 564 & 19,1 & 196 & 20,1 & 165 & 16,5 \\
\hline \multicolumn{7}{|l|}{ Nível de Habilitação Parental ${ }^{1}$} \\
\hline Ensino Básico & 915 & 31,3 & 286 & 29,5 & 318 & 32,1 \\
\hline Ensino Secundário & 1114 & 38,1 & 384 & 39,7 & 381 & 38,5 \\
\hline Ensino Superior & 897 & 30,7 & 298 & 30,8 & 291 & 29,4 \\
\hline \multicolumn{7}{|l|}{ Nacionalidade } \\
\hline Portugal & 2697 & 91,6 & 876 & 90,6 & 913 & 91,2 \\
\hline Outro país & 246 & 8,4 & 91 & 9,4 & 88 & 8,8 \\
\hline \multicolumn{7}{|l|}{ Religião } \\
\hline Católica & 1875 & 81,9 & 617 & 81,0 & 617 & 79,1 \\
\hline Outra religião Cristã & 92 & 4,0 & 33 & 4,3 & 37 & 4,7 \\
\hline Ateísmo / Agnosticismo & 293 & 12,8 & 103 & 13,5 & 113 & 14,5 \\
\hline Outra & 29 & 1,3 & 9 & 1,2 & 13 & 1,7 \\
\hline \multicolumn{7}{|l|}{ Descrição étnica } \\
\hline Branco/a ou Caucasiano/a & 2563 & 88,8 & 845 & 88,7 & 863 & 87,4 \\
\hline Negro/a & 248 & 8,6 & 84 & 8,8 & 93 & 9,4 \\
\hline Mestiço/a ou Moreno/a & 44 & 1,5 & 11 & 1,2 & 23 & 2,3 \\
\hline Outra & 30 & 1,0 & 13 & 1,4 & 8 & 0,8 \\
\hline \multicolumn{7}{|l|}{ Orientação Sexual } \\
\hline Heterossexual & 2841 & 97,1 & 937 & 97,2 & 958 & 96,3 \\
\hline Bissexual & 70 & 2,4 & 24 & 2,5 & 29 & 2,9 \\
\hline Gay/Lésbica & 14 & 0,5 & 3 & 0,3 & 8 & 0,8 \\
\hline
\end{tabular}

Nota. ${ }^{1} \mathrm{O}$ nível de habilitação parental corresponde ao nível de formação mais elevado obtido por um dos progenitores.

de defesa dos direitos da população LGBT: "As pessoas agem como se houvesse algo de errado contigo" e "As pessoas tratam-te de forma negativa por acharem que te pareces com uma pessoa do sexo oposto". Para assegurar a validade facial, o instrumento foi alvo de uma reflexão falada junto de seis jovens que frequentavam os dois tipos de ensino - científico-humanístico e profissional - e em anos escolares diferentes, no âmbito da qual foi sugerida a explicitação das instruções para a consequente seleção do principal "motivo" para a discriminação.

Os participantes foram convidados a responder aos 11 itens (9 originais mais 2 criados para o estudo) numa escala de resposta de tipo Likert de 6-pontos: 0 - nunca; 1 -raramente (menos de 1 vez/ano), 2 - por vezes (algumas vezes/ano), 3 - algumas vezes (algumas vezes/mês), 4 - muitas vezes (pelo menos uma vez/semana) e 5 -quase 
sempre (quase todos os dias). Caso os participantes indicassem algum item com uma frequência igual ou superior a 2 (por vezes; algumas vezes/ano), eram convidados a selecionar apenas uma característica que considerassem ser o principal motivo para o tratamento injusto (nacionalidade, sexo, etnia ou raça, idade, religião, altura, peso, outro aspeto da aparência física, orientação sexual, nível económico ou educacional, condição ou problema físico, condição ou problema mental, ou outro motivo). Dado que as características não são mutuamente exclusivas, os resultados revelam que $27,8 \%$ dos respondentes selecionou várias dimensões caracterizadoras (e.g., nacionalidade, idade, peso, nível económico), algo que também se tinha observado no estudo de Kessler et al. (1999). Em termos de percentagem seguiu-se a menção a um "outro" motivo (23,6\%; e.g., sobretudo aspectos relacionados à personalidade ou a conflitos com colegas), aparência física (12,1\%), idade $(11,11 \%)$, peso $(6 \%)$, etnia ou raça $(4,2 \%)$, altura $(4,0 \%)$ nível económico ou educacional $(3,8 \%)$, nacionalidade $(2,3 \%)$, orientação sexual $(2,0 \%)$, religião $(1,0 \%)$, condição física $(1,0 \%)$, sexo ou gênero $(0,8 \%)$ e condição mental $(0,5 \%)$.

Inventário de Saúde Mental. Considerado o impacto negativo da percepção da discriminação na saúde mental (e.g., Schmitt et al., 2014) explorou-se a relação da escala adaptada com o instrumento Mental Health Inventory-5 (Ribeiro, 2001; Veit \& Ware, 1983). Os cinco itens (e.g., "Durante o último mês com que frequência te sentiste: Triste e em baixo?"; item revertido) foram avaliados numa escala tipo Likert de 5-pontos - desde 1 (nunca) a 5 (quase sempre). $\mathrm{Na}$ análise fatorial confirmatória, o modelo foi re-especificado de modo a permitir duas correlações entre três erros de mensuração. Esta modificação, para além de permitir um incremento considerável no ajustamento global do modelo, é admissível do ponto de vista teórico. A primeira foi entre os erros de mensurações dos itens "muito nervoso/a" (item revertido) e "calmo/a e em paz" e justifica-se por ambas as manifestações serem influenciados pelo sistema nervoso autónomo (simpático e parassimpático). A segunda correlação foi entre os erros dos itens "calmo/a e em paz" e "uma pessoa feliz" e justifica-se por ambos reportarem a avaliação positiva do estado emocional da pessoa. O ajustamento final do modelo foi considerado aceitável $\left(\chi^{2} / g l=4,156\right.$; CFI $=$ 0,$998 ;$ RMSEA $=0,032 ; p_{\text {RSMEA }}=0,927$; SRMR $=0,008$; $\lambda>0,540$; Cronbach $\alpha=0,834$ ).

\section{Procedimentos}

Coleta de Dados. O estudo foi aprovado pelo Comitê de Ética da Faculdade de Psicologia e de Ciências da Educação da Universidade do Porto, pela Comissão Nacional de Proteção de Dados e recebeu permissão do Ministério da Educação para ser conduzido em estabelecimentos de ensino públicos em Portugal. Em cinco cidades dos dois maiores centros urbanos de Portugal (Grande Lisboa e Grande Porto), 24 escolas colaboraram no estudo. Encar- regados de educação e alunos foram informados sobre o principal objetivo do estudo - como os jovens lidam com situações sociais negativas -, o procedimento e a confidencialidade dos dados e deram o seu consentimento à participação. Os alunos preencheram o questionário em contexto de sala de aula na presença da primeira autora deste estudo. A taxa de participação foi de 94,7\%.

Análise dos Dados. Para avaliar a validade fatorial da escala, começou-se por uma análise da distribuição dos resultados e pela consideração dos valores absolutos de assimetria e curtose $(\mathrm{sk}<2, \mathrm{ku}<7)$. $\mathrm{Na}$ análise fatorial exploratória (AFE), foi utilizado o método de fatoração de eixo principal com rotação oblíqua e normalização de Kaiser. Para apreciação dos resultados, considerou-se o valor da estatística Kaiser-Meyer-Olkin (KMO > 0,7), o teste de esfericidade de Bartlett $(p<0,05)$ e os pesos fatoriais dos itens $(\lambda>0,32)$. Para avaliação da consistência interna de cada fator, analisou-se o alfa de Cronbach $(\alpha>$ 0, 7; Costello \& Osborne, 2005; Hair, Anderson, Tatham, \& Black, 2005; Marôco, 2011). Estas análises foram efetuadas no software SPSS (v.22).

Com recurso ao software AMOS (v.22) realizou-se a análise fatorial confirmatória (AFC), utilizando o método da máxima verosimilhança. Procedeu-se à imputação dos valores omissos por via de regressão, com base no padrão de respostas nos itens da escala. Na maioria dos itens, observaram-se um ou dois casos de valores omissos, com exceção do item 7 (ver Tabela 2) no qual se observaram quatro casos omissos. Para apreciação da qualidade do ajustamento do modelo, consideraram-se os valores do qui-quadrado ajustado $\left(X^{2} / g l<0,5\right)$, do Bentler Comparative Fit Index (CFI $>0,90)$, do Root Mean Square Error of Approximation (RMSEA $<0,08 ; p<0,05$ ) e do Standardized Root Mean Square Residual (SRMR $<0,08$ ), em consonância com as indicações citadas em Marôco (2010). Os índices de modificação sugeridos pelo AMOS foram utilizados para reformulações ao modelo. Para aprofundamento da aferição da validade de constructo da escala, foram realizadas: (a) comparações de médias, em função da raça/etnia e orientação sexual dos participantes, utilizando o teste estatístico MANOVA com testes post hoc Games-Howell (por este não requerer homogeneidade de variâncias e por se estar a comparar grupos com tamanhos de amostra diferentes; Marôco, 2011), e (b) correlações de Pearson entre a escala adaptada e o instrumento Mental Health Inventory-5.

\section{Resultados}

Considerando os valores de assimetria e curtose, observou-se que o item criado para o estudo "As pessoas tratam-te de forma negativa por acharem que te pareces com uma pessoa do sexo oposto" apresentou uma distribuição leptocúrtica ( $\mathrm{sk}=3,772 ; \mathrm{ku}=15,461$ ) o que justifica a sua exclusão das análises posteriores. Dado que os restantes itens não apresentaram grandes desvios na sua distribuição, 
Freitas, D. F., Coimbra, S., Marturano, E. M. \& Fontaine, A. M. (2015). Adaptação da Escala de Discriminação Quotidiana para Jovens Portugueses.

a AFE foi conduzida com os outros dez itens. A análise dos indicadores à validade da $\mathrm{AFE}(\mathrm{KMO}=0,816$; teste de esfericidade de Bartlett $p<0,001$ ) revelou que esta análise é adequada para esta escala nesta amostra. Inicialmente, a configuração obtida sugeria uma solução de três fatores com uma variância total explicada de 59,09\%. Porém, a análise das comunalidades revelou que dois itens apresentaram valores bastante baixos: (a) "Em lojas, restaurantes ou noutros serviços és menos bem atendido/a do que as outras pessoas" $\left(h^{2}=0,067\right)$; (b) "As pessoas agem como se tivessem medo de ti" $\left(h^{2}=0,210\right)$. Estes itens foram excluídos das análises e uma segunda AFE foi conduzida. Os resultados revelaram uma solução de dois fatores com uma variância total explicada de $57,29 \%$. Na solução de dois fatores todos os itens apresentaram pesos fatoriais satisfatórios $(\lambda>0,499)$. Considerando que, em análises prévias à estrutura da escala, já havia sido sugerida uma estrutura bifatorial (Barnes et al., 2004; Guyll et al., 2001), considerou-se a presente organização fatorial adequada. Os itens do primeiro fator traduziam comportamentos de tratamento diferenciado e abertamente ofensivos. Tendo como referência a terminologia já utilizada por Barnes et al. (2004), este fator foi nomeado de Tratamento Injusto. Os itens do segundo fator traduziam uma depreciação mais camuflada da pessoa, sendo este fator designado de Rejeição Pessoal. Ambos os fatores apresentaram valores de consistência interna satisfatórios $(\mathrm{F} 1-\alpha=0,747$; F2 $-\alpha=0,736)$.

Tabela 2

Resultados da Análise Fatorial Exploratória à Escala de Discriminação Quotidiana

Fatores

Itens

Tratamento Injusto Rejeição Pessoal

$h^{2}$
1. És tratado/a com menos respeito do que as outras pessoas.

2. És tratado/a com menos simpatia do que as outras pessoas.

0,760

0,682

$\mathbf{0 , 5 7 8}$

9. És ameaçado/a ou provocado/a.

8. Chamam-te nomes ou insultam-te.

4. As pessoas agem como se pensassem que não és inteligente.

5. As pessoas agem como se houvesse algo de errado contigo.

6. As pessoas agem como se fossem melhores do que tu.

7. As pessoas agem como se pensassem que és desonesto/a. $\mathbf{0 , 5 3 8}$

$-0,116$

0,100

0,100

0,037

3,474
0,053

0,040

$-0,055$

0,052

0,790

0,705

$\mathbf{0 , 5 0 3}$

0,499

1,110
Variância explicada

$57,29 \%$
Foi conduzida uma análise fatorial confirmatória (AFC) para testar a solução fatorial obtida na análise exploratória: dois fatores correlacionados, com quatro itens cada. Considerando que a solução fatorial obtida na AFE inclui sete itens da escala original e um item elaborado no âmbito deste estudo, de forma a conseguir a maior equivalência possível com a métrica da escala original, a AFC desenvolveu-se em dois passos. No primeiro passo, procedeu-se à estimação de um modelo com dois fatores correlacionados, contendo apenas os sete itens originais. Partindo dos valores de regressão de cada item estimados neste modelo, no segundo passo, procedeu-se à aferição do ajustamento do modelo com os dois fatores e oito itens, tendo-se fixado os parâmetros dos sete itens originais de acordo com os valores de regressão obtidos no primeiro modelo. Neste modelo todos os itens apresentaram pesos fatoriais satisfatórios $(\lambda>0,521)$ e os fatores apresentavam uma correlação entre si elevada $(r=0,701)$. $O$ ajustamento global do modelo revelou-se, contudo, insatisfatório $\left(\chi^{2} / g l=12,943 ; \mathrm{CFI}=0,881\right.$; PCFI $=0,755$; RMSEA $=$ 0,$\left.109 ; p_{\text {RSMEA }}<0,001 ; \mathrm{SRMR}=0,061 ; \mathrm{AIC}=350,638\right)$. Considerando a alta correlação entre os dois fatores, e os resultados pouco favoráveis do modelo com dois fatores, decidiu-se testar um modelo de segunda ordem: um fator geral, a Percepção da Discriminação, e dois fatores específicos, o Tratamento Injusto e a Rejeição Pessoal. Este modelo revelou um índice de ajustamento satisfatório, após a re-especificação do mesmo, incorporando quatro correlações entre os erros de mensuração dos seguintes itens: (a) dos itens 8 e 9 (IM = 112,382), (b) dos itens 4 e $5(\mathrm{IM}=32,270),(\mathrm{c})$ dos itens 4 e $6(\mathrm{IM}=31,050)$, e (d) dos itens 2 e 1 (IM $=15,181$; ver descrição dos itens na Tabela 2). É de sublinhar que nos casos (a), (b), (d), os itens foram apresentados de forma contígua no questionário e 
em alguns casos corresponderam a construções frásicas similares e que remetem para uma dimensão próxima. No caso (c), a introdução da correlação considerou-se adequada, dado que não ser considerado "inteligente" poderá estar também associado a que outras pessoas ajam como se "fossem melhores". Obtiveram-se os seguintes índices de ajustamento global: $\chi^{2} / g l=3,548$; CFI $=0,979$; RM-
$\mathrm{SEA}=0,050 ; p_{\mathrm{RSMEA}}=0,467 ; \mathrm{SRMR}=0,033 ; \lambda>0,464$. Observa-se que a Percepção de Discriminação explica 88\% da variância do fator Tratamento Injusto $(\beta=0,941)$ e $78 \%$ da variância do fator Rejeição Pessoal $(\beta=0,885)$, fatores que, por sua vez, explicam de $22 \%$ a $60 \%$ da variância das respostas nos itens. Na Figura 1, observam-se os valores de regressão estandardizados e a variância explicada das variáveis do modelo final.

\section{AFC - Escala de Discriminação Quotidiana $X 2(20)=70,956 ; p=, 000 ; X 2 / g l=3,548$ $\mathrm{CFI}=, 979$; RMSEA $=, 050 ; \mathrm{p}(\mathrm{rmsea})=, 467$}

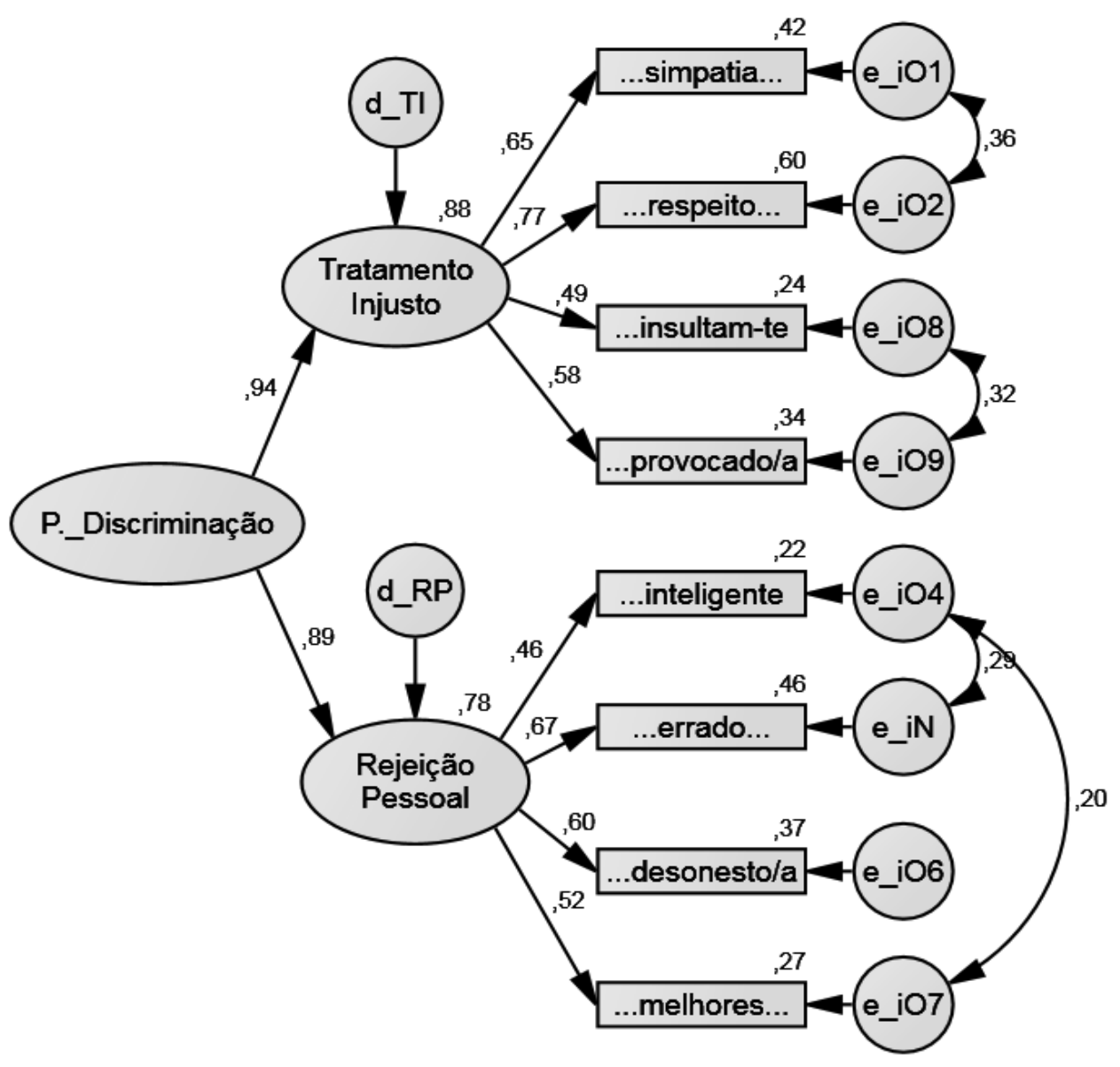

Figura 1. Modelo hierárquico da Escala de Discriminação Quotidiana.

No que concerne à recolha de elementos que suportam a validade de constructo do instrumento, a comparação de médias por via de MANOVAS revelou um efeito principal da raça/etnia dos participantes no nível de discriminação percepcionada $[$ Traço de Pillai $=0,008, F(6,5734)=4,068$, $\left.p<0,001, \eta_{p}{ }^{2}=0,004, \pi=0,977\right]$. Este efeito foi significativo nos níveis de Rejeição Pessoal $[F(3,2867)=6,094$, $p<0,001]$, sendo que a média apresentada por pessoas negras $(M=1,314 ; D P=1,046)$ é significativamente maior do que a das pessoas brancas $(M=1,050 ; D P=0,967)$, sendo estas diferenças de pequena magnitude ( $d$ de Cohen $=0,262)$. Os valores apresentados por pessoas mestiças ( $M$ $=1,279 ; D P=1,218)$ ou com outra identificação étnica $(M$ $=1,058 ; D P=0,928)$ não diferem significativamente dos demais. Já as médias de Tratamento Injusto apresentadas por pessoas brancas $(M=0,866 ; D P=0,869)$, negras $(M$ $=0,935 ; D P=0,864)$, mestiças $(M=1,000 ; D P=1,026)$ ou com outra identificação étnica $(M=1,083 ; D P=0,963)$ não são significativamente diferentes $[F(3,2867)=1,337$, $p=0,260]$. A orientação sexual também exerceu um efeito 
Freitas, D. F., Coimbra, S., Marturano, E. M. \& Fontaine, A. M. (2015). Adaptação da Escala de Discriminação Quotidiana para Jovens Portugueses.

principal nos níveis de discriminação percepcionada [Traço de Pillai $=0,004, F(2,2908)=5,493, p=0,004, \eta_{p}{ }^{2}=0,004$, $\pi=0,852]$. Os níveis de Tratamento Injusto $[F(1,2909)$ $=10,986, p=0,001]$ dos participantes LGB $(M=1,182$; $D P=1,081)$ são significativamente mais elevados do que os de pessoas heterossexuais $(M=0,863 ; D P=0,862)$, sendo estas diferenças de pequena magnitude ( $d$ de Cohen $=0,326$ ). Similarmente, na dimensão de Rejeição Pessoal $[F(1,2909)=4,506, p=0,034]$, observa-se uma diferença de pequena magnitude $(d$ de Cohen $=0,224)$ entre a média dos participantes LGB $(M=1,298 ; D P=1,078)$ e a dos participantes heterossexuais $(M=1,068 ; D P=0,975)$.

As análises correlacionais revelam que, como previsto, há uma associação destes fatores com a saúde mental: uma correlação negativa moderada foi observada entre o nível de Saúde Mental e a subescala de Tratamento Injusto $\left[r(2960)=-0,335, p<0,001 ; r^{2}=0,112\right]$ e a subescala de Rejeição Pessoal $\left[r(2960)=-0,379, p<0,001 ; r^{2}=0,143\right]$.

\section{Discussão}

O procedimento de adaptação e validação do instrumento da Escala de Discriminação Quotidiana envolveu a tradução dos itens da escala original, inclusão de itens para aumento da amplitude de comportamentos avaliados, a aferição da validade facial junto de especialistas e de jovens estudantes, a medição da validade fatorial exploratória e confirmatória e da consistência interna. Estes procedimentos de validação contribuem para a validade ecológica e de constructo do instrumento (cf. Borsboom et al., 2004; Tanzer \& Sim, 1999).

A estrutura fatorial obtida na análise exploratória é constituída por dois fatores, Tratamento Injusto e Rejeição Pessoal, cada um composto por quatro itens (destes, sete itens são da escala original e um foi elaborado no âmbito da adaptação). As dimensões fatoriais obtidas apresentaram níveis de consistência interna satisfatórios. Com a realização da AFC verificou-se que a organização fatorial mais apropriada para o instrumento corresponde a um modelo hierárquico de segunda ordem, no qual as as variáveis latentes de primeira ordem Tratamento Injusto e Rejeição Pessoal estão subordinados à dimensão latente de segunda ordem, Percepção de Discriminação. Os resultados da AFC revelam um ajustamento do modelo com ajustamento global satisfatório, sustentando a validade fatorial da escala adaptada. A introdução de correlações entre erros de mensuração foi considerada apropriada, atendendo a que a contiguidade da apresentação dos itens (tal como na versão original), poderá ter aumentado a tendência para respostas semelhantes. De forma a minimizar esta tendência as autoras propõem uma reordenação na apresentação dos itens da escala. Para aceder à proposta elaborada no âmbito desta adaptação, sugere-se o contacto com as autoras.

Uma configuração fatorial de dois fatores já havia sido apresentada em outros estudos (Barnes et al., 2004; Guyll et al., 2001). Contudo, existem diferenças que vale a pena sublinhar. Por comparação à estrutura apresentada por Barnes et al., (2004), observa-se que: (a) os itens 8 e 9 saturavam no fator "rejeição pessoal" e (b) o item 6 saturava em ambos os fatores, porém com maior peso no fator "tratamento injusto". Por comparação à estrutura apresentada por Guyll et al. (2001), as principais diferenças observadas são: (a) o item 7 saturava no fator "discriminação flagrante" e (b) os itens 1 e 2 saturam no fator "discriminação subtil". Estes dados parecem sugerir alguma variação da estrutura da escala em diferentes amostras, em função de diferenças regionais, culturais e, possivelmente, em função da faixa etária da amostra. No entanto, apenas um estudo transcultural e transversal a várias faixas etárias permitiria esclarecer cabalmente, quais as principais fontes de variação contextual na manifestação do constructo.

A escala demonstra, ainda, ter sensibilidade discriminante entre grupos, no sentido previsto: observaram-se diferenças nos níveis de Tratamento Injusto entre participantes LGB e heterossexuais e nos níveis de Rejeição Pessoal entre participantes LGB e heterossexuais e também entre participantes negros e brancos. Estes dados constituem fontes adicionais de validade de constructo do instrumento avaliado (Borsboom et al., 2004), ao corroborarem a superioridade de perceção de discriminação entre pessoas LGB e pessoas negras (e.g., Pascoe \& Richman, 2009). No entanto, há que ressaltar a baixa magnitude do efeito, assim como a ausência de diferenças ao nível do Tratamento Injusto em função da raça/etnia. Considerando que no estudo de Barnes et al. (2004) observou-se que os participantes afro-americanos negros experienciaram níveis maiores de Tratamento Injusto do que os brancos, cremos que a não confirmação da nossa hipótese de estudo esteja mais relacionada com o efeito da cultura ocidental - que inibe comportamentos abertamente racistas e caracteriza-se por novas formas de expressão deste preconceito (e.g., França \& Monteiro, 2004; Pereira, Raquel, \& Torres, 2003; Pereira \& Vala, 2007) - do que com a inadequação da escala para avaliar situações de discriminação. Estudos centrados na expressão atual do preconceito revelam uma tendência para as pessoas discriminarem pessoas negras apenas quando o contexto fornece alguma justificação para tal, tais como a adequação às expectativas da população dominante (Pereira et al., 2003), o pior desempenho numa tarefa (França $\&$ Monteiro, 2004) ou a ativação da norma de meritocracia (Pereira \& Vala, 2007). Adicionalmente, sabe-se que esta adaptação das manifestações do racismo ao contexto social é desenvolvida durante a infância (França \& Monteiro, 2004). Deste modo, na adolescência já há uma inibição de comportamentos abertamente racistas (como os que constituem os itens do fator Tratamento Injusto). Contudo, a atitude negativa perante pessoas negras é manifestada por comportamentos mais encobertos, o que justifica o maior nível de percepção de Rejeição Pessoal apresentado pelos jovens negros da amostra. O preconceito contra pessoas homossexuais ainda é considerado normativo, pelo que 
tal inibição social parece não existir perante pessoas LGB (França \& Monteiro, 2004).

À semelhança do que foi anteriormente observado (Barnes et al., 2004; Kessler et al., 1999; Taylor et al., 2004), os fatores extraídos no modelo fatorial Tratamento Injusto e Rejeição Pessoal relacionam-se negativamente com a saúde mental, estando associados a $11 \%$ e $14 \%$, respectivamente, da variação nesta dimensão. Em conjunto, estes dados sublinham o efeito negativo da discriminação e apoiam a validade de constructo do instrumento.

No que concerne às limitações do presente estudo, um dos constrangimentos observados relaciona-se com a amplitude etária da nossa amostra, constituída apenas por estudantes de escolas secundárias. Reconhecemos que a adaptação da escala para uma amostra de adultos ou idosos poderá levar a uma estruturação diferente da mesma, pois os contextos de interação das pessoas são mais diversificados na idade adulta (e.g., variados locais e tipos de trabalho), aumentando também o reportório de experiências sociais (positivas e negativas). Um dos pontos fortes da Escala de Discriminação Quotidiana é, exatamente, a possibilidade de avaliação da percepção de tratamento injusto em função de uma grande diversidade de condições. Uma forma de garantir um maior rigor entre as comparações realizadas é a avaliação da equivalência do modelo estrutural nesses diferentes grupos. Este procedimento pode ser realizado através de uma Análise Fatorial Confirmatória Multigrupos (cf. Marôco, 2010). Não nos foi possível conduzir tal análise dada a heterogeneidade da amostra e a relativamente baixa frequência de (a) jovens de minorias étnicas ou sexuais e (b) participantes que selecionaram apenas uma característica-motivo para a discriminação. Reconhecemos, no entanto, que este é um aspecto relevante para uma validação mais rigorosa da escala e consideramos que deverá ser abordado em estudos futuros.

\section{Conclusão}

A versão adaptada da Escala de Discriminação Quotidiana, pela validade de constructo e ecológica demonstrada, revela ser um recurso apropriado para a avaliação e comparação da percepção de discriminação quotidiana em jovens. Embora esta escala tenha sido utilizada (e adaptada) a populações que diferem sobretudo em termos raciais/étnicos, os itens são abrangentes e ela é passível de capturar a percepção do tratamento injusto tendo por base diversas motivações. $\mathrm{O}$ instrumento permite conhecer a frequência destas vivências e a sua associação a diversas características, nomeadamente algumas não tão abordadas na literatura (e.g., peso, doença mental ou incapacidade física; Schmitt et al., 2014). Considerada a relação da discriminação com a saúde mental e física e com dimensões psicossociais (ver Pascoe \& Richman, 2009; Schmitt et al., 2014), cremos que o instrumento poderá ser utilizado em situações de avaliação em intervenções ao nível indi- vidual e coletivo (e.g., comunidade, escola ou empresa). Concluímos que a Escala de Discriminação Quotidiana, estruturada em dois fatores, é apropriada para aferir este fenômeno junto de jovens portugueses.

\section{Referências}

Barnes, L. L., de Leon, C. F. M., Wilson, R. S., Bienias, J. L., Bennett, D. A., \& Evans, D. A. (2004). Racial differences in perceived discrimination in a community population of older blacks and whites. Journal of Aging and Health, 16(3), 315337. doi:10.1177/0898264304264202

Borsboom, D., Mellenbergh, G. J., \& van Heerden, J. (2004). The concept of validity. Psychological Review, 111(4), 1061-1071. doi:10.1037/0033-295X.111.4.1061

Costello, A. B., \& Osborne, J. W. (2005). Best practices in exploratory factor analysis: Four recommendations for getting the most from your analysis. Practical Assessment, Research \& Evaluation, 10(7), 1-9.

D’Augelli, A. R., Grossman, A. H., \& Starks, M. T. (2008). Gender atypicality and sexual orientation development among lesbian, gay, and bisexual youth. Journal of Gay \& Lesbian Mental Health, 12(1-2), 121-143. doi:10.1300/ J529v12n01 08

Dovidio, J., Major, B., \& Crocker, J. (2000). Stigma: Introduction and overview. In T. Heatherton, R. Kleck, M. Hebl, \& J. Hull (Eds.), The social psychology of stigma (pp. 1-30). New York: The Guildford Press.

França, D. X., \& Monteiro, M. B. (2004). A expressão das formas indirectas de racismo na infância. Análise Psicológica, 22(4), 705-720.

Gato, J., Carneiro, N. S., \& Fontaine, A. M. (2011). Contributo para uma revisitação histórica e crítica do preconceito contra as pessoas não heterossexuais. Crítica e Sociedade: Revista de Cultura Politica, 1(1), 139-167.

Goffman, E. (1990). Stigma: Notes on the management of a spoiled identity. London: Penguin Books. (Originalmente publicado em 1963)

Guyll, M., Matthews, K. A., \& Bromberger, J. T. (2001). Discrimination and unfair treatment: Relationship to cardiovascular reactivity among African American and European American women. Health Psychology, 20(5), 315-325. doi:10.1037//0278-6133.20.5.315

Hair, J., Anderson, R., Tatham, R., \& Black, W. (2005). Análise multivariada de dados (5. ed.). São Paulo, SP: Bookman.

Kessler, R. C., Mickelson, K. D., \& Williams, D. R. (1999). The prevalence, distribution, and mental health correlates of perceived discrimination in the United States. Journal of Health and Social Behavior, 40(3), 208-230.

Kim, G., Sellbom, M., \& Ford, K. L. (2014). Race/ethnicity and measurement equivalence of the Everyday Discrimination Scale. Psychological Assessment, 26(3), 892-900. doi: $10.1037 / \mathrm{a} 0036431$

Krieger, N. (1999). Embodying inequality: A review of concepts, measures, and methods for studying health consequences of discrimination. International Journal of Health Services, 29(2), 295-352. doi:10.2190/M11W-VWXE-KQM9-G97Q

Krieger, N., Smith, K., Naishadham, D., Hartman, C., \& Barbeau, E. M. (2005). Experiences of discrimination: Validity and reliability of a self-report measure for population health research on racism and health. Social Science \& Medicine, 61, 1576-1596. doi:10.1016/j.socscimed.2005.03.006 
Freitas, D. F., Coimbra, S., Marturano, E. M. \& Fontaine, A. M. (2015). Adaptação da Escala de Discriminação Quotidiana para Jovens Portugueses.

Lewis, T. T., Yang, F. M., Jacobs, E. A., \& Fitchett, G. (2012). Racial/ethnic differences in responses to the everyday discrimination scale: A differential item functioning analysis. American Journal of Epidemiology, 175(5), 391-401. doi:10.1093/aje/kwr287

Major, B., \& Sawyer, P. (2009). Attributions to discrimination: Antecedents and consequences. In T. D. Nelson (Ed.), Handbook of prejudice, stereotyping, and discrimination (pp. 89-93). New York: Psychology Press.

Marôco, J. (2010). Análise de equações estruturais. Fundamentos teóricos, software \& aplicações. Pêro Pinheiro, Portugal: Report Number.

Marôco, J. (2011). Análise estatística com o SPSS Statistics (5. ed.). Pêro Pinheiro, Portugal: Report Number.

Pascoe, E., \& Richman, L. (2009). Perceived discrimination and health: A meta-analytic review. Psychological Bulletin, 135(4), 531-554. doi:10.1037/a0016059

Pereira, C., Raquel, A., \& Torres, R. (2003). Um estudo do preconceito na perspectiva das representações sociais: Análise da Influência de um discurso justificador da discriminação no preconceito racial. Psicologia: Reflexão e Crítica, 16(1), 95-107.

Pereira, C., \& Vala, J. (2007). Preconceito, normas sociais e justificações para a discriminação das pessoas negras. In M. B. Monteiro, M. M. Calheiro, R. Jerónimo, C. Mouro, \& P. Duarte (Eds.), Percursos da investigação em Psicologia Social e Organizacional (Vol. 2, pp. 145-164). Lisboa, Portugal: Colibri.

Ribeiro, J. L. P. (2001). Mental Health Inventory: Um estudo de adaptação à população portuguesa. Psicologia, Saúde \& Doenças, 2(1), 77-99.

Richman, L. S., \& Leary, M. R. (2009). Reactions to discrimination, stigmatization, ostracism, and other forms of interpersonal rejection: A multimotive model. Psychological Review, 116(2), 365-383. doi:10.1037/a0015250

Schmitt, M. T., Branscombe, N., Postmes, T., \& Garcia, A. (2014). The consequences of perceived discrimination for psychological well-being: A meta-analytic review. Psychological Bulletin, 140(4), 921-948. doi:10.1037/a0035754

Seaton, E. K., Caldwell, C. H., Sellers, R. M., \& Jackson, J. S. (2010). An intersectional approach for understanding perceived discrimination and psychological well-being among African American and Caribbean Black youth. Developmental Psychology, 46(5), 1372-1379. doi:10.1037/a0019869

Smedley, A., \& Smedley, B. D. (2005). Race as biology is fiction, racism as a social problem is real: Anthropological and historical perspectives on the social construction of race. American Psychologist, 60(1), 16-26. doi:10.1037/0003-066X.60.1.16

Stucky, B. D., Gottfredson, N. C., Panter, A. T., Daye, C. E., Allen, W. R., \& Wightman, L. F. (2011). An item factor analysis and item response theory-based revision of the Everyday Discrimination Scale. Cultural Diversity and Ethnic Minority Psychology, 17(2), 175-185. doi:10.1037/a0023356

Tanzer, N. K., \& Sim, C. Q. E. (1999). Adapting instruments for use in multiple languages and cultures: A review of the ITC Guidelines for Test Adaptations. European Journal of Psychological Assessment, 15(3), 258-269. doi:10.1027//10155759.15.3.258

Taylor, T. R., Kamarck, T. W., \& Shiffman, S. (2004). Validation of the Detroit Area Study Discrimination Scale in a community sample of older African American adults: The Pittsburgh healthy heart project. International Journal of Behavioral Medicine, 11(2), 88-94. doi:10.1207/s15327558ijbm1102_4
Veit, C. T., \& Ware, J. E. (1983). The structure of psychological distress and well-being in general populations. Journal of Consulting and Clinical Psychology, 51(5), 730-742. doi:10.1037/0022-006X.51.5.730

Williams, D. R., \& Mohammed, S. A. (2009). Discrimination and racial disparities in health: Evidence and needed research. Journal of Behavioral Medicine, 32(1), 20-47. doi:10.1007/ s10865-008-9185-0

Williams, D. R., Yu, Y., Jackson, J. S., \& Anderson, N. B. (1997). Racial differences in physical and mental health. Journal of Health Psychology, 2(3), 335-351. 